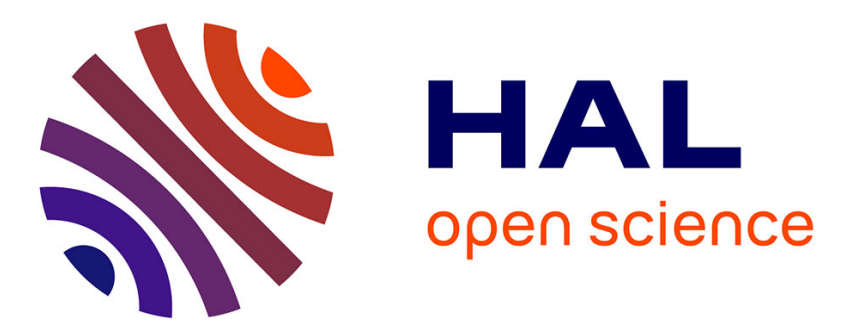

\title{
An Adaptive Simulation Framework for the Exploration of Extreme and Unexpected Events in Dynamic Engineered Systems
}

\author{
Pietro Turati, Nicola Pedroni, Enrico Zio
}

\section{- To cite this version:}

Pietro Turati, Nicola Pedroni, Enrico Zio. An Adaptive Simulation Framework for the Exploration of Extreme and Unexpected Events in Dynamic Engineered Systems. Risk Analysis, 2017, 37 (1), pp.147 - 159. 10.1111/risa.12593. hal-01652286

\section{HAL Id: hal-01652286 \\ https://hal.science/hal-01652286}

Submitted on 30 Nov 2017

HAL is a multi-disciplinary open access archive for the deposit and dissemination of scientific research documents, whether they are published or not. The documents may come from teaching and research institutions in France or abroad, or from public or private research centers.
L'archive ouverte pluridisciplinaire HAL, est destinée au dépôt et à la diffusion de documents scientifiques de niveau recherche, publiés ou non, émanant des établissements d'enseignement et de recherche français ou étrangers, des laboratoires publics ou privés. 


\section{An adaptive simulation framework for the efficient, semi-automatic exploration of extreme and unexpected events in the...}

Article in Risk Analysis · January 2017

DOI: $10.1111 /$ risa.12593

CITATION

1

3 authors:

Pietro Turati

CentraleSupélec

9 PUBLICATIONS 16 CITATIONS

SEE PROFILE

Enrico Zio

Politecnico di Milano

835 PUBLICATIONS 9,735 CITATIONS

SEE PROFILE
READS

66
Nicola Pedroni

Politecnico di Torino

103 PUBLICATIONS 629 CITATIONS

SEE PROFILE

Some of the authors of this publication are also working on these related projects:

Project Qualitative information extraction using classification-based methods View project

Advanced computational methods for modelling the mechanisms of degradation in equipments of

Project electricity production plants and uncertainty modelling and propagation View project 


\title{
An Adaptive Simulation Framework for the Exploration of Extreme and Unexpected Events in Dynamic Engineered Systems
}

\author{
Pietro Turati, ${ }^{1}$ Nicola Pedroni, ${ }^{1}$ and Enrico Zio ${ }^{1,2, *}$
}

\begin{abstract}
The end states reached by an engineered system during an accident scenario depend not only on the sequences of the events composing the scenario, but also on their timing and magnitudes. Including these additional features within an overarching framework can render the analysis infeasible in practical cases, due to the high dimension of the system state-space and the computational effort correspondingly needed to explore the possible system evolutions in search of the interesting (and very rare) ones of failure. To tackle this hurdle, in this article we introduce a framework for efficiently probing the space of event sequences of a dynamic system by means of a guided Monte Carlo simulation. Such framework is semi-automatic and allows embedding the analyst's prior knowledge about the system and his/her objectives of analysis. Specifically, the framework allows adaptively and intelligently allocating the simulation efforts preferably on those sequences leading to outcomes of interest for the objectives of the analysis, e.g., typically those that are more safety-critical (and/or rare). The emerging diversification in the filling of the state-space by the preference-guided exploration allows also the retrieval of critical system features, which can be useful to analysts and designers for taking appropriate means of prevention and mitigation of dangerous and/or unexpected consequences. A dynamic system for gas transmission is considered as a case study to demonstrate the application of the method.
\end{abstract}

KEY WORDS: Dynamic event tree; integrated deterministic probabilistic safety assessment (IDPSA); Markov Chain Monte Carlo (MCMC); random exploration; unexpected accident scenarios

\section{INTRODUCTION}

The dynamic response of an engineered system under different conditions can be studied, in general, by means of mathematical models implemented in corresponding computer codes for numerical simulations. In particular, in the analysis of safety-critical

${ }^{1}$ Chair System Science and the Energy Challenge, Fondation Electricité de France (EDF), CentraleSupélec, Université ParisSaclay, Chatenay-Malabry, France.

${ }^{2}$ Energy Department, Politecnico di Milano, Milan, Italy.

*Address correspondence to Enrico Zio Chair System Science and the Energy Challenge, Fondation Electricité de France (EDF), CentraleSupélec, Université Paris-Saclay, Grande Voie des Vignes,92290, Chatenay-Malabry, France; enrico.zio@polimi.it, enrico.zio@centralesupelec.fr. systems, such as nuclear power plants, oil and gas facilities, electrical grids, etc. model simulations are used to identify extreme configurations and avoid that they remain unexplored and unknown until their (possibly catastrophic) occurrence. ${ }^{(1-3)}$ The outputs of the simulations guide the analysis of the system evolutions and the identification of those event sequences (i.e., scenarios) that can lead the system into extreme conditions. ${ }^{(4-6)}$ In this context, the combination of event trees (ETs) (representing the logic of the system) and mathematical models of the system dynamics (describing the dynamics of the physical phenomena involved) has been largely advocated as the way for determining the end states (ESs) that can be reached by the system and 
deriving the corresponding causality relations among the events. ${ }^{(7-9)}$

In this line of thought, works on dynamic event trees (DETs) ${ }^{(10-15)}$ have highlighted that the end states reached by a system as a result of an accident scenario do not depend only on the order of occurrence of the events in the sequence, but also on the exact time at which these events occur and on their magnitude..$^{8,9,16-18)}$ However, the introduction of the time and magnitude dimensions into the analysis makes the size of the system state-space theoretically infinite and, thus, impossible to be explored completely. Also (and in any case), the computational time needed for running a single simulation of the system evolution can be significant: consider, for example, the computer code RELAP used to simulate the thermo-hydraulic behavior of nuclear systems, which can take hours or days for a single run in specific conditions. ${ }^{(19-21)}$ To address this issue, the majority of the methods available in the literature exploit the discretization of the time dimension and the pruning of ET branches that have low probability, for the purpose of reducing the number of possible event sequences to be explored; however, these techniques may miss "rare" sequences leading to extreme safetycritical outcomes, as pointed out in Refs. 15 and 22, where the authors consider the possibility of biasing the exploration toward critical events.

In view of these challenges, efficient methods for integrated deterministic probabilistic safety assessment (IDPSA) are currently being developed to take into account time dependences in the evolution of the dynamic system and to probe the corresponding event sequence space for identifying unknown unreliability, unexpected scenarios, and critical configurations. ${ }^{(23)}$ Along this line of research, this article contributes an efficient framework for exploring the state-space of a dynamic system with the purpose of discovering event sequences leading to unexpected outcomes. In this context, Refs. 24 and 25 propose methods that focus the exploration efforts, i.e., the simulations, on those scenarios having more uncertain outcomes (i.e., a higher number of end states). For this purpose, they exploit a function based on negative entropy for assessing the uncertainty in the outcomes and a Bayesian scheme for updating the knowledge gathered by the simulations. ${ }^{(26-28)}$ As a result, scenarios that can reach a larger number of ESs are explored more frequently and thoroughly.

In this article, new driving functions are proposed to allow embedding the analyst's knowledge and preferences into the exploration, e.g., the interest in specific scenarios or ESs. The new driving functions are implemented within a novel, adaptive, semi-automatic exploration framework for efficiently probing the system state-space and retrieving the corresponding information of interest. Demonstration is given with regards to a simple, but representative, dynamic system made by a gas transmission pipe actively controlled by a valve and connected in series to two other pipes in parallel. All the system components are subject to stochastic failures, described by assigned probability distributions. The results of the method are compared to those of a crude Monte Carlo sampling and an entropy-based search scheme of the system state-space. ${ }^{(25)}$

The rest of the article is organized as follows. In Section 2, a general description of the problem under analysis is given. In Section 3, the semi-automatic adaptive exploration framework is presented. In Section 4, the case study and the associated results are reported. Finally, in Section 5, some conclusions are drawn and prospective research challenges are suggested.

\section{PROBLEM DEFINITION}

Within the framework of interest of the present article, a scenario defines a specific sequence (i.e., order) of events in the life evolution of the dynamic system, which may involve a particular group of components, safety functions, or actions (e.g., mechanical failures, activation of safety systems, and human decisions). In this context, DET is used as the logical modeling technique to derive, by means of simulation, the scenarios that can arise in the life evolution of a dynamic system as the result of a sequence of successes and failures of different components and functions. ${ }^{(8,29)}$ Much software for DET analyses, such as DYLAM, ${ }^{(10)}$ ADS, ${ }^{(11)}$ and MCDET, ${ }^{(14)}$ is available and, in principle, all the possible (accident) scenarios could be extracted, especially by recurring to massive parallel computing ${ }^{(30)}$ or to backtracking techniques. ${ }^{(8)}$ However, not all the possible different time sequences within a given scenario can be explored, by reason of the extremely high computational cost needed for simulating all of them. This is relevant since, as shown, for example, in Refs. 18 and 31, different time sequences (even within the same scenario) may lead to different outcomes, hereafter also called end states (ESs). This justifies the research interests and efforts in taking into account the time dimension of the problem. Typically, 
an end state is a categorical variable representing in a synthetic way the configuration of the system, e.g., the degradation level of the components (i.e., low, medium, and high degradation) and the state of the system (safe, warning, failure). For the sake of clarity, let us consider a component that starts deteriorating once an initiating event (damage) occurs. Then, the state of degradation of the component, i.e., its end state $E S$, at a given mission time $T_{\text {Miss }}$ depends on the time $T_{\text {init }}$ of the initiating damage. An illustrative example is reported in Fig. 1.

However, depending on the number and typology of the events involved in a scenario, the impact of the event times on the system model simulations can be different. In this light, some scenarios may always lead to similar simulations generating the same outcome; on the contrary, other sequences can lead to a larger variability in the outcomes. For this reason, during the exploration of the possible system evolutions, in some cases, it may be in the analyst's interest to focus the simulation efforts on those scenarios that show higher variability in the ESs (i.e., that have more uncertain outcomes). To this aim, a novel approach, which takes its root in Ref. 25, is here proposed. The general procedure consists of two steps: (i) selection of a scenario that is "worth" being explored according to a predefined "driving criterion"; and (ii) simulation of a possible system evolution, conditioned to the selected scenario.

In other situations, instead, the analyst may be already aware of some possible evolutions and he/she may be interested in precisely identifying those time sequences belonging to a given scenario $S_{j}$ that can lead to a particular $E S_{i}$, with the objective of retrieving information and features of the scenario useful to prevent them and prepare for protection against and mitigation of their consequences. ${ }^{(18,29)}$

Since the contribution of the article mainly lies in the efficient and intelligent exploration of the system state-space, with no interest in the estimation of the probabilities of the events of interest, in all the situations considered (see above) we propose to sample the components' transition times from a joint distribution uniform on the support defined by the scenario selected. ${ }^{(25)}$ For the sake of clarity, let us consider a scenario $S_{j}$ that involves the change of states of two components, namely, $A$ and $B$, in a specific order within the mission time (e.g., in this case $T_{A}<$ $\left.T_{B} \leq T_{\text {Miss }}\right)$. Then, in order to simulate one possible system evolution, we sample the couple $\left(T_{A}, T_{B}\right)$ from the joint uniform distribution on the support defined by $S_{j}$, which is shown in Fig. 2 as a shaded region. For this purpose, we resort to a MCMC Gibbs sampling. ${ }^{(32)}$

By resorting to a uniform distribution, a more thorough exploration of the time window under analysis is obtained. Actually, the occurrences of component failures are frequently modeled by exponential distributions; this implies that the likelihood of the time of occurrence of an event decreases with the increment of its value. This makes some system evolutions and, hence, some ESs extremely unlikely to occur, thus reducing the exploration capability of a method based on plain random sampling from those (original) distributions. In this respect, Fig. 3 shows the probability density function of the time $T_{\text {int }}$ of

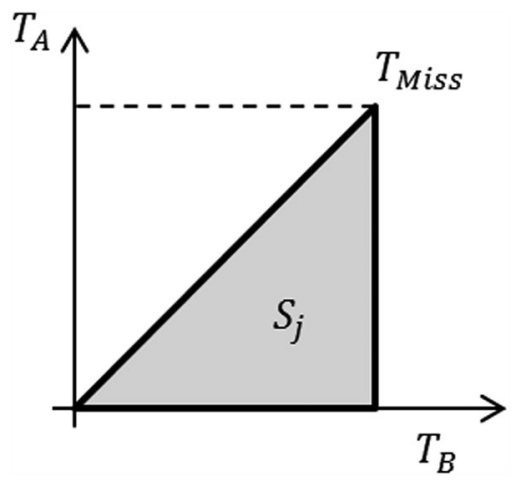

Fig. 2. Support of the time of occurrence of events $A$ and $B$ defining scenario $S_{j}$, where $T_{A}<T_{B} \leq T_{\text {Miss }}$.
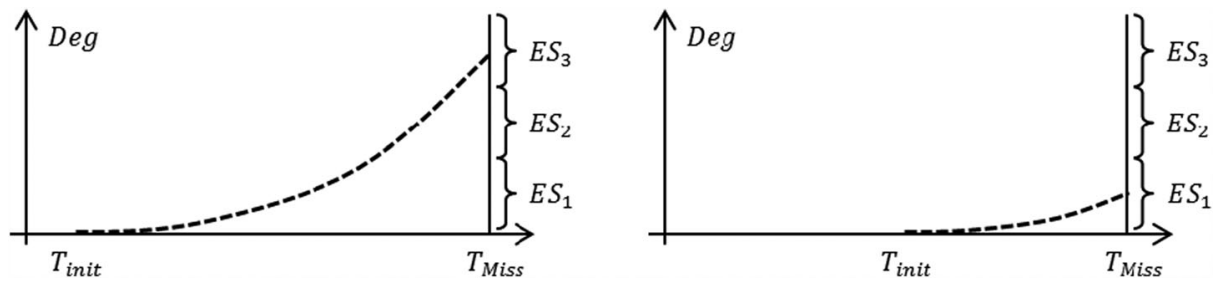

Fig. 1. Illustrative example of the effects that different damage initiating times $T_{\text {int }}$ have on the final degradation state of a fictitious component. 


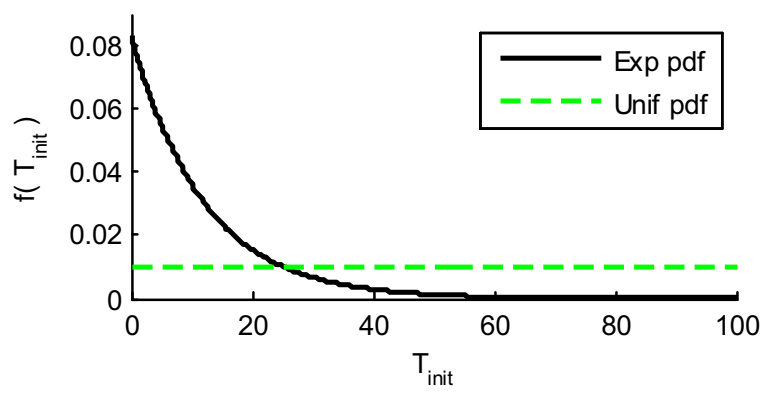

Fig. 3. Exponential pdf (dark line) and uniform pdf (light dashed line) defined on the support $\left[0, T_{\text {Miss }}=100\right]$

the initiating event introduced in the trivial example above, in the cases of an exponential conditional distribution with support defined on [0, $\left.T_{\text {Miss }}=100\right]$ and of a uniform distribution defined on the same support.

\section{A NOVEL FRAMEWORK FOR THE SEMI-AUTOMATIC ADAPTIVE EXPLORATION OF THE STATE-SPACE OF DYNAMIC SYSTEMS}

The semi-automatic adaptive exploration method consists of three main steps, as sketched in the flow diagram of Fig. 4:

(1) preliminary exploration (Section 3.1), i.e., a global exploration of the whole space of the dynamic system according to two possible driving criteria, namely, the guided (Section 3.1.1) and the forced (Section 3.1.2) exploration; this step aims at enhancing the general knowledge of the analyst regarding the role and impact of different time sequences in the evolution of the dynamic system;

(2) analyst decision (Section 3.2), i.e., after the preliminary exploration, the analyst can decide to either improve his/her global view of the state space by increasing the number of simulations according to the criteria of the preliminary exploration (step 1), or focus

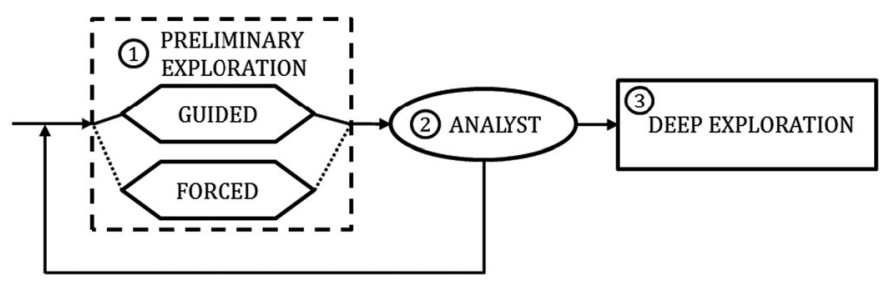

his/her attention on a specific event of interest (step 3);

(3) deep exploration (Section 3.3), i.e., a thorough exploration of a particular event: for example, the objective can be that of retrieving the possible evolutions within the scenario $S_{j}$ that can reach a given end state $E S_{i}$, indicated hereafter as the pair $\left\{S_{j}, E S_{i}\right\}$.

\subsection{Preliminary Exploration}

The preliminary exploration aims at efficiently retrieving information about the general dynamic behavior of the system (model) under the constraint of limited computational effort (i.e., of a fixed number of available simulations to run): in other words, for each scenario $S_{j}$, it aims at identifying all the ESs that it can "generate." For this purpose, the analyst can choose either a preliminary guided exploration (Section 3.1.1) or a preliminary forced exploration (Section 3.1.2) according to his/her interest and to the information already available.

\subsubsection{Guided Exploration}

If the analyst does not have any prior information about the outcomes of the system accident scenarios or if he/she has scarce information, but he/she is not interested in any specific outcome based on the current knowledge at his/her disposal, a "guided exploration" framework should be chosen. In particular, the choice of the scenario that will be explored through a new simulation run is automatically made by selecting the scenario $S^{*}$ which maximizes the driving function $I_{\gamma}(\cdot)$ :

$$
S^{*}=\underset{j \in S}{\operatorname{argmax}} I_{\gamma}\left(S_{j}\right),
$$

where $I_{\gamma}\left(S_{j}\right)$ is defined as:

$$
I_{\gamma}\left(S_{j}\right)=I_{\gamma}\left(n_{j}, \quad N_{j}^{E S}\right)=\frac{\left(N_{j}^{E S}\right)^{\gamma}}{n_{j}},
$$

Fig. 4. General flow diagram of the exploration framework proposed. 
Table I. Average Results of 1,000 Experiments

\begin{tabular}{rrrrrrrrrrrrrrrr}
\hline & $E S_{1}$ & $E S_{2}$ & $E S_{3}$ & $E S_{4}$ & Tot & $E S_{1}$ & $E S_{2}$ & $E S_{3}$ & $E S_{4}$ & Tot & $E S_{1}$ & $E S_{2}$ & $E S_{3}$ & $E S_{4}$ & $T$ ot \\
\hline$S_{1}$ & 47.9 & 0.0 & 0.0 & 0.0 & 47.9 & 25.0 & 0.0 & 0.0 & 0.0 & 25.0 & 10.0 & 0.0 & 0.0 & 0.0 & 10.0 \\
$S_{2}$ & 12.0 & 11.9 & 0.0 & 0.0 & 23.9 & 12.4 & 12.6 & 0.0 & 0.0 & 25.0 & 10.0 & 9.9 & 0.0 & 0.0 & 20.0 \\
$S_{3}$ & 5.3 & 5.3 & 5.3 & 0.0 & 15.9 & 8.4 & 8.3 & 8.3 & 0.0 & 25.0 & 10.0 & 10.0 & 10.0 & 0.0 & 30.0 \\
$S_{4}$ & 3.0 & 3.1 & 3.0 & 3.1 & 12.2 & 6.2 & 6.2 & 6.3 & 6.3 & 25.0 & 10.0 & 10.0 & 9.9 & 10.1 & 40.0 \\
\hline
\end{tabular}

Note: Each experiment runs 100 simulations of preliminary guided exploration with different values of the parameter $\gamma:-1$ (left); 0 (middle); 1 (right). Column "Tot" represents the total number of simulations run within the respective scenario.

where $N_{j}^{E S}$ is the number of ESs that scenario $S_{j}$ can reach (if this information is not available, then it represents the number of ESs that have already been visited within the scenario and it is updated when a new ES is discovered by a new simulation run); $n_{j}$ is the number of simulations that have already been run within $S_{j} ; \gamma \in(-\infty,+\infty)$ is a parameter that should be chosen according to the preference of the analyst: $(i)$ if $\gamma<0$, the driving function chooses more frequently those scenarios that can reach a small number of ESs; (ii) if $\gamma=0$, no preference is given to any scenario; (iii) if $\gamma>0$, the driving function selects more likely those scenarios that can reach a large number of ESs. For the sake of clarity and by way of example, consider a simple dynamic system where only four scenarios can occur $S_{1}, \ldots, S_{4}$ and where each scenario can reach a different number of end states, $N_{1}^{E S}=1, \quad N_{2}^{E S}=2, \ldots, N_{4}^{E S}=4$. Finally, let us assume that all reachable ESs in the same scenario have the same probability of occurring. Table I reports the average of 1,000 explorations, performed with 100 simulations each, that have been distributed among the different scenarios according to three different values of the parameter $\gamma$, i.e., $\gamma=-1$ (left), $\gamma=0$ (middle), and $\gamma=1$ (right).

The choice of parameter $\gamma=1$ is particularly suitable because, in this case, the exploration algorithm distributes the simulations among all the scenarios in order to guarantee that each scenario $S_{j}$ "gathers" a number of simulations proportional to the number $N_{j}^{E S}$ of end states that each scenario can "generate."

\subsubsection{Forced Exploration}

As in the preliminary guided exploration, the algorithm selects the scenario $S^{*}$ that maximizes a given driving function and, then, simulates a dynamic evolution conditioned to the selected scenario $S^{*}$. However, the driving function, namely, $I_{\beta}(\cdot)$, of the preliminary forced exploration is defined as:

$$
\begin{aligned}
I_{\beta}\left(S_{j}, E S^{*}\right) & =I_{\beta}\left(n_{j}, \quad N_{j}^{E S}, I_{E S^{*}}\right) \\
& = \begin{cases}\frac{\left(N_{j}^{E S}\right)^{\gamma}}{n_{j}}, & I_{E S^{*}}=0 \\
\frac{\left(N_{j}^{E S}\right)^{\gamma}}{n_{j}}, \beta, & I_{E S^{*}}=1\end{cases}
\end{aligned}
$$

where $E S^{*}$ is a particular set of end states of interest for the analyst; $I_{E S^{*}}$ is a Boolean variable, which equals 1 if the simulations of scenario $S_{j}$ can reach at least one of the end states in $E S^{*}$, and 0 otherwise; $\beta \in(0,+\infty)$ is the forcing parameter: the higher it is, the more frequently the algorithm selects those scenarios that can reach the end states in $E S^{*}$; finally, the remaining variables are defined as in Equation (2). With respect to the case of the preliminary exploration, a different number of ESs can be included in the set $E S^{*}$, i.e., the cardinality of $E S^{*}$ can be larger than 1 .

For the sake of clarity, let us consider the same trivial example introduced above (Table I), assume parameter $\gamma=1$, and suppose that we are interested in gathering information about those scenarios that can reach end states $E S^{*}=\left\{E S_{3} ; E S_{4}\right\}$ (e.g., because they represent extremely dangerous conditions). Table II reports the effects of different choices of parameter $\beta=\{0.25 ; 1 ; 4\}$ on the final distribution of the simulation runs among the scenarios. If $\beta \in$ $(0,1)$, those scenarios that can reach the set $E S^{*}$ are penalized in the selection step (left); if $\beta=1$, the algorithm turns to the preliminary guided exploration described above (middle); finally, if $\beta \in(1,+\infty)$, the scenarios that can reach the set $E S^{*}$ are favored in the selection step (right).

For the preliminary forced exploration, we have proposed only one function based on one single parameter beta, which reflects an interest about a set of known end states; however, a variety of functions could be used at this stage to force the selection of scenarios according to other desirable criteria. 
Table II. Average Results of 1,000 Experiments

\begin{tabular}{|c|c|c|c|c|c|c|c|c|c|c|c|c|c|c|c|}
\hline & $E S_{1}$ & $E S_{2}$ & $E S_{3}$ & $E S_{4}$ & Tot & $E S_{1}$ & $E S_{2}$ & $E S_{3}$ & $E S_{4}$ & Tot & $E S_{1}$ & $E S_{2}$ & $E S_{3}$ & $E S_{4}$ & Tot \\
\hline$S_{1}$ & 21.0 & 0.0 & 0.0 & 0.0 & 21.0 & 10.0 & 0.0 & 0.0 & 0.0 & 10.0 & 3.1 & 0.0 & 0.0 & 0.0 & 3.1 \\
\hline$S_{2}$ & 21.1 & 21.0 & 0.0 & 0.0 & 42.1 & 10.0 & 9.9 & 0.0 & 0.0 & 20.0 & 3.0 & 2.9 & 0.0 & 0.0 & 5.9 \\
\hline$S_{3}$ & 5.3 & 5.5 & 5.1 & 0.0 & 16.0 & 10.0 & 10.0 & 10.0 & 0.0 & 30.0 & 12.6 & 12.3 & 12.5 & 0.0 & 37.4 \\
\hline$S_{4}$ & 5.4 & 5.1 & 5.1 & 5.2 & 20.9 & 10.0 & 10.0 & 9.9 & 10.1 & 40.0 & 13.4 & 13.4 & 13.4 & 13.3 & 53.5 \\
\hline
\end{tabular}

Note: Each experiment run 100 simulations of preliminary forced exploration with $\gamma=1$ and with different values of the parameter $\beta: 0.25$ (left); 1 (middle); 4 (right). Column "Tot" represents the total number of simulations run within the respective scenario.

\subsection{Analyst Decision}

Every time a preliminary exploration is performed, matrices, such those reported in Table I and Table II, become available. Hence, based on the events visited (i.e., on the pairs scenario-end state $\left.\left\{S_{j}, E S_{i}\right\}\right)$ and on the number of simulations that have been run to visit them, the analyst can decide either to increase the number of simulations according to the criteria adopted in the preliminary exploration phase or to perform a deeper and more refined exploration of specific events of interest. According to his/her preference, the analyst has to iteratively choose the maximum allowable number of simulations that can be run according to the preliminary or deep exploration, respectively. In many cases, the dimension of the system (state-space) and the variability of its behavior (in practice, the number of ESs a scenario can reach and the corresponding probabilities), are not known a priori; on the contrary, the computational cost needed for a system simulation can be known (e.g., in terms of average time per simulation). Then, the computational effort can be considered as a constraint that the analyst needs to take into account in accordance with his/her preferences among the different exploration criteria. In this respect, it must be noticed that the proposed method does not guarantee that the whole event space is probed: inevitably, if the computational capacity available (in practice, the total number of simulations that can be run) is small compared to the size of the system state-space, only a limited number of end states can be explored for each scenario.

\subsection{Deep Exploration}

The objective of the deep exploration is to identify, as precisely as possible, which system evolutions (i.e., in practice which combinations of transition times) can lead to a given event of interest. For the sake of clarity, we assume that an event of in- terest is defined as the pair $\{$ Scenario-End-State $\}=$ $\left\{S_{j}, E S^{*}\right\}$; nonetheless, with no loss of generality $E S^{*}$ can represent also an ensemble of end states. Given the structure of the mathematical model, the guiding idea of the deep exploration is to generate time sequences "around" those that have already reached the event $\left\{S_{j}, E S^{*}\right\}$. In order to achieve this goal, we resort to a Markov Chain Monte Carlo (MCMC) method, which allows to generate a set of random samples from any desired (namely, target) probability distribution $p(\cdot){ }^{(32)}$ In detail, we utilize a MetropolisHasting (M-H) algorithm ${ }^{(33)}$ to sample components' transition times uniformly on the support $S E S^{*}$ of the event of interest $\left\{S_{j}, E S^{*}\right\}$, in other words, to sample uniformly among the transition times that lead to the event of interest $\left\{S_{j}, E S^{*}\right\}$. The M-H algorithm consists of two steps: $(i)$ proposition of a new candidate $\boldsymbol{T}^{*}$ (in this case, a vector of transition times) in accordance to a proposal distribution $q(\cdot)$; and (ii) acceptance or rejection of the proposed time vector. For the first step, we utilize as proposal a multivariate Gaussian distribution $q\left(\boldsymbol{T}^{*} \mid \boldsymbol{T}_{n}\right) \sim$ $N\left(\boldsymbol{T}_{n}, \Sigma\right)$, having as mean value the last vector of transition times $\boldsymbol{T}_{n}$ accepted in the region of interest and as covariance matrix $\Sigma$. In particular, $\Sigma$ is estimated using the set of transition times generated during the preliminary exploration that have led the system to the event of interest. This choice of the covariance matrix provides a "favorable" acceptance ratio $(A R)$ between the number of proposed and accepted values. Regarding the second step, the proposed value $\boldsymbol{T}^{*}$ is accepted (i.e., $\boldsymbol{T}_{n+1}=$ $\boldsymbol{T}^{*}$ ) or rejected (i.e., $\boldsymbol{T}_{n+1}=\boldsymbol{T}_{n}$ ) with a probability $\alpha\left(\boldsymbol{T}_{n}, \boldsymbol{T}^{*}\right)=\min \left(r\left(\boldsymbol{T}_{n}, \boldsymbol{T}^{*}\right), 1\right)$, where $r$ is defined as follows:

$$
r\left(\boldsymbol{T}_{n}, \boldsymbol{T}^{*}\right)=\left\{\begin{array}{cl}
\frac{p\left(\boldsymbol{T}^{*}\right) q\left(\boldsymbol{T}_{n} \mid \boldsymbol{T}^{*}\right)}{p\left(\boldsymbol{T}_{n}\right) q\left(\boldsymbol{T}^{*} \mid \boldsymbol{T}_{n}\right)}, & p\left(\boldsymbol{T}_{n}\right) q\left(\boldsymbol{T}^{*} \mid \boldsymbol{T}_{n}\right)>0 \\
1, & \text { otherwise }
\end{array}\right.
$$

$p(\cdot)$ being the target distribution from which we want to sample. Thanks to the symmetry of the Gaussian 
proposal $q\left(\boldsymbol{T}_{n} \mid \boldsymbol{T}^{*}\right)=q\left(\boldsymbol{T}^{*} \mid \boldsymbol{T}_{n}\right)$, then, Equation (4) can be rewritten as:

$$
r\left(\boldsymbol{T}_{n}, \boldsymbol{T}^{*}\right)=\left\{\begin{array}{c}
\frac{p\left(\boldsymbol{T}^{*}\right)}{p\left(\boldsymbol{T}_{n}\right)}, p\left(\boldsymbol{T}_{n}\right)>0[3 p t] \\
1, \text { otherwise. }
\end{array}\right.
$$

Finally, since the target distribution is uniform on the support $S E S^{*}$ of the event of interest, then, the probability $\alpha\left(\boldsymbol{T}_{n}, \boldsymbol{T}^{*}\right)$ can be written as:

$$
\alpha\left(\boldsymbol{T}_{n}, \boldsymbol{T}^{*}\right)= \begin{cases}1, & \boldsymbol{T}^{*} \in S E S^{*} \\ 0, & \text { otherwise. }\end{cases}
$$

However, in order to know whether the proposed occurrence time vector $\boldsymbol{T}^{*}$ belongs to $S E S^{*}$, we need to verify that: $(a) \boldsymbol{T}^{*}$ satisfies the time order characterizing scenario $S_{j} ;(b)$ adds to the end state of interest $E S^{*}$. It is worth verifying the two conditions in the mentioned order, since the second condition implies a system simulation run and the corresponding computational cost.

It must be kept in mind that, whenever the M-H algorithm is used, the acceptance ratio $(A R)$ between the proposed samples and the accepted ones plays a fundamental role. Too high acceptance ratios $(A R>$ $0.9)$ are a symptom of a proposal $q(\cdot)$ with too small variability, i.e., most of the proposed $\boldsymbol{T}^{*}$ are too close to the original ones and, thus, the algorithm results too slow in probing the support $S E S^{*}$; on the contrary, too small acceptance ratios $(A R<0.2)$ are a symptom of a proposal $q(\cdot)$ with too high variability, i.e., most of the proposed $\boldsymbol{T}^{*}$ are likely to fall out of the support of interest $S E S^{*}$. In this respect, adaptive MCMC methods exploiting an adaptive proposal distribution have been presented in the literature and can be employed at this stage to "optimally" fill the support $S E S^{*}$ of interest. ${ }^{(34,35)}$ Nonetheless, if the majority of the proposed samples are rejected due to the constraint $(a)$ of belonging to a given scenario (i.e., of presenting a given precise event timing), an MCMC Gibbs sampler (G-S), such as the one introduced in Section 2, may be useful. Actually, the G$\mathrm{S}$ allows sampling occurrence time vectors that implicitly satisfy the time order constraint $(a)$, probing the entire scenario instead of the support $S E S^{*}$ only. Thus, roughly speaking, on the basis of the "area" of the support of the scenario $S_{j}$ that is covered by the end state $E S^{*}$ of interest, the analyst can choose different MCMC methods: if the portion of transition time vectors that leads to $E S^{*}$ is small (Fig. 5, right), an M-H algorithm should be preferred to fill this "small portion" of scenario $S_{j}$; on the contrary, if the portion of transition time vectors leading to $E S^{*}$
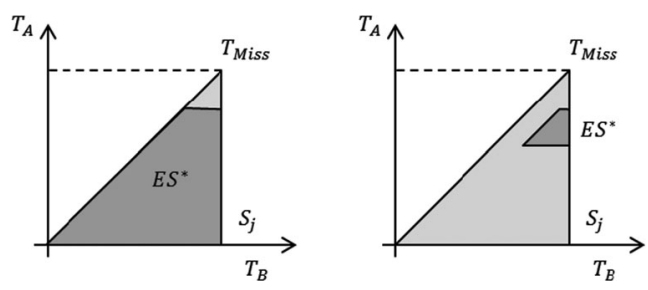

Fig. 5. The dark area represents the portion of scenario $S_{j}$ that leads to the end state of interest $E S^{*}$.

is large (Fig. 5, left), G-S should be preferred, in order to put more effort on the "even coverage" of the entire scenario $S_{j}$.

Regarding the approach used to choose the number of simulations to run for performing the deep exploration, two criteria are proposed: $(i)$ fixed number of simulations (as in the preliminary exploration of Section 3.1); (ii) level of filling of the support of the event of interest. For what concerns the second criterion, the idea is to keep on generating new simulation outcomes until the support of the event of interest is filled by an amount of points (i.e., configurations) that "sufficiently" cover the entire outcome variability. In detail, after the preliminary exploration a set of occurrence time vectors $E X_{V}\left(S E S^{*}\right)=\left\{\boldsymbol{T}_{1}, \ldots, \boldsymbol{T}_{V}\right\}$ that lead to the event of interest $\left\{S_{j}, E S^{*}\right\}$ is available. As a measure of the (time) space filling, the maximum of the minimum distances among these time vectors is considered: then, a time filling index $D_{V}\left(E X_{V}\left(S E S^{*}\right)\right)$ after the preliminary exploration is computed as:

$$
D_{V}\left(E X_{V}\left(S E S^{*}\right)\right)=\max _{i \in E X_{V}\left(S E S^{*}\right)} \min _{j \neq i} d\left(\boldsymbol{T}_{i}, \boldsymbol{T}_{j}\right)
$$

where $d(\cdot, \cdot)$ represents a proper distance between two vectors. In this article, for example, we consider the Euclidean one. Whenever a new time vector $\boldsymbol{T}_{n}$ is accepted during the exploration, it is added to the set of time vectors that lead to the event of interest, i.e., $E X_{n}\left(S E S^{*}\right)=\left\{E X_{n-1}\left(S E S^{*}\right) ; \boldsymbol{T}_{n}\right\}$, and the filling index $D_{n}\left(E X_{n}\left(S E S^{*}\right)\right)$ is consequently updated. The deep exploration ends when the ratio between the current filling index and the preliminary one falls below a fixed threshold $\delta \in[0,1]$, i.e., when the "density" of time vectors in the support $S E S^{*}$ of interest is $\sim(1 / \delta)^{l}$ times higher than the preliminary one, being $l$ the size of the time vector $\boldsymbol{T}_{n}$. Nonetheless, a maximum allowable number $n_{\max }$ of samples is also set, in order to limit in any case the maximum 
Table III. Sketch of the Algorithm Describing the Deep Exploration Stopping Criterion

1. For $i=1, \ldots, V$ evaluate the minimum distances from the vector $T_{i}$ and save them in the vector $d_{V}$ : $d_{V}(i)=\min _{j \neq i} d\left(T_{i}, T_{j}\right)$. According to this notation $D_{V}\left(E X_{V}\left(S E S^{*}\right)\right)=\max d_{V}$.

2. Given a new time vector $T_{n}$, update the $d_{n-1}$ vector for $i=1, \ldots, n-1$ : $d_{n}(i)=\min \left(d_{n-1}(i), d\left(T_{i}, T_{n}\right)\right)$,

3. Add the $n$th component to $d_{n-1}$ recurring to the distance already available from the previous step: $d_{n}(n)=\min _{j \neq n} d\left(T_{n}, T_{j}\right)$.

4. Evaluate the filling index: $D_{n}\left(E X_{n}\left(S E S^{*}\right)\right)=\max d_{n}$.

5. Check if the stopping criteria are satisfied: $\frac{D_{n}\left(E X_{n}\left(S E S^{*}\right)\right)}{D_{V}\left(E X_{V}\left(S E S^{*}\right)\right)}<\delta$ or $n>n_{\max }$ If not, return to step 2 .

computational effort. Then, the stopping criterion becomes:

$$
\frac{D_{n}\left(E X_{n}\left(S E S^{*}\right)\right)}{D_{V}\left(E X_{V}\left(S E S^{*}\right)\right)}<\delta \text { or } n>n_{\max } .
$$

The corresponding algorithm is summarized in Table III.

The space filling capability of the algorithm is strictly related to the dimension of the vectors involved: in practice, the higher the dimension, the larger the number of random vectors needed to reduce the filling index.

\section{CASE STUDY}

The case study under analysis is a gas transmission subnetwork composed of two pipes in parallel and another one in series. The input of each pipe is controlled by a valve. The whole block diagram is shown in Fig. 6, where each pair valve-pipe is considered as a single block.

Each pipe can transmit gas with a maximum flow rate of $\left[\phi_{a}, \phi_{b}, \phi_{c}\right]=[8,5,5] \cdot 10^{4} \mathrm{~m}^{3} /$ day, for pipes $a, b, c$, respectively. A control system adjusts

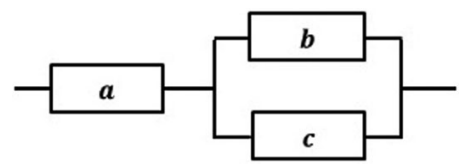

Fig. 6. Block diagram of the system under analysis.

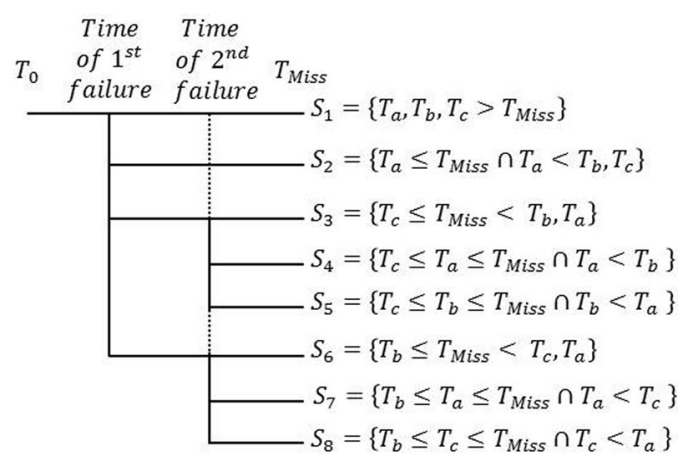

Fig. 7. Event tree representation of the eight scenarios that can occur, where $T_{a}, T_{b}, T_{c}$ are the times of failures of components $a$, $b, c$, respectively, and $T_{\text {Miss }}$ is the mission time.

the opening of the valves in order to guarantee the equilibrium between the input and output flows. Fig. 7 shows the ET containing all the scenarios that can occur in the system. If one of the pipes in parallel breaks, the control system immediately closes the corresponding valve and increases the flow rate of the remaining pipe to the maximum, in order to compensate for the diminished flow. No reparation strategies are considered. The system presents eight possible scenarios with different operating conditions: (i) safe, i.e., all pipes are functioning correctly; (ii) overloaded, i.e., one of the pipes in parallel is closed; (iii) broken, i.e., no gas is provided by the system.

The ESs for each scenario have been defined and classified on the basis of two output variables: (i) the amount of gas provided in safe conditions (GSC), i.e., when all the components are functioning correctly; (ii) the amount of gas provided in overloaded conditions (GOC), i.e., when one of the two pipes in parallel is down and the remaining one works at its maximum flow rate. With respect to that, $\mathrm{GSC}_{\max }$ and $\mathrm{GOC}_{\max }$ indicate the maximum quantities of gas that can be provided within the mission time $T_{\text {Miss }}=900 \mathrm{~d}$, in safe and overloaded conditions, respectively, i.e., GS $C_{\max }=\phi_{\mathrm{a}} \cdot T_{\text {Miss }}$ and $\mathrm{GO} C_{\max }=\max \left(\phi_{\mathrm{b}}, \phi_{\mathrm{c}}\right) \cdot T_{\text {Miss }}$. The ESs are, then, divided into six classes according to the criteria reported in Fig. 8. For example, $\mathrm{E}_{4}=\left\{\frac{1}{3} \mathrm{GSC}_{\max }<\mathrm{GSC} \leq \frac{2}{3} \mathrm{GSC}_{\max } \cap 0 \leq \mathrm{GOC} \leq\right.$ $\left.\frac{1}{3} \mathrm{GOC}_{\max }\right\}$, which means that the system has operated for a medium period of time in safe conditions $\left(\frac{1}{3} \mathrm{GSC}_{\max }<\mathrm{GSC} \leq \frac{2}{3} \mathrm{GSC}_{\max }\right)$ and, then, once it goes in overloaded conditions, it breaks down $\left(0 \leq \mathrm{GOC} \leq \frac{1}{3} \mathrm{GOC}_{\max }\right)$.

It must be noticed that not all the ESs can be reached by all scenarios: Table IV (left matrix) 
reports those ESs that can be reached by a given scenario (indicated by 1) and those that cannot (indicated by 0): each column in the table represents an ES and each row represents a scenario. This information is usually not available $a$ priori and in general its retrieval represents one of the objectives of the state-space exploration. However, it is used here to analyze the performance of the proposed method. In Table IV (middle and right), two additional matrices show the ESs reachable for two sets of different gas flow rates, e.g., $\left[\phi_{a}, \phi_{b}, \phi_{c}\right]=[8,3.7,5] \cdot 10^{4} \mathrm{~m}^{3} /$ day and $\left[\phi_{a}, \phi_{b}, \phi_{c}\right]=[8,2.2,6] \cdot 10^{4} \mathrm{~m}^{3} /$ day, respectively. These values have been chosen in order to analyze the performance of the method for different parameters values, which implies that the number of reachable ESs varies.

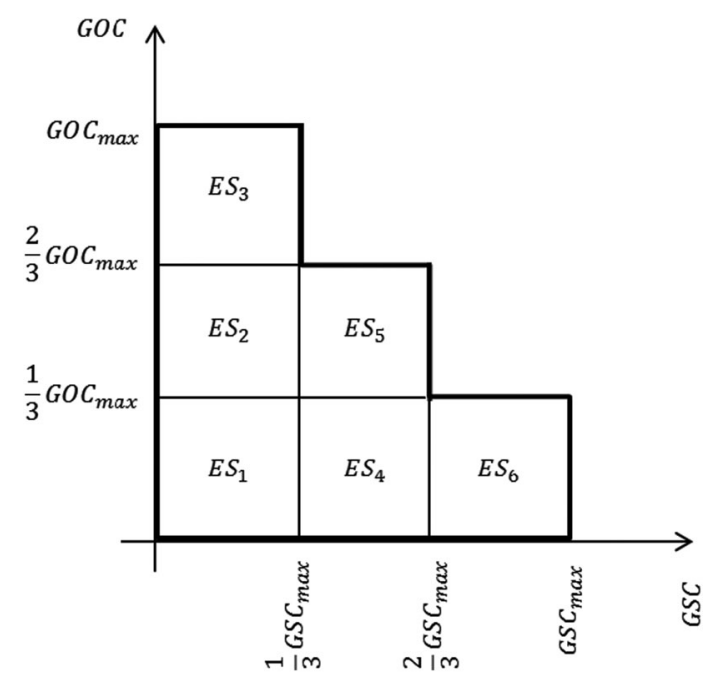

Fig. 8. Classification of the end states (ESs) according to the two output variables GSC and GOC.

\subsection{Preliminary Guided Exploration}

To evaluate the performance of the preliminary guided exploration, two indices are introduced: (i) the number of simulations needed for the first complete exploration (NFE), i.e., the number of simulations that should be run to visit at least once all the reachable ESs for all the scenarios; (ii) the number of simulations needed for the second complete exploration (NSE), i.e., the number of simulations that should be run to visit all the reachable ESs for all the scenarios at least twice. NFE gives information about the number of simulations needed to explore all the events defined by the pairs \{Scenario, EndState $\}=\{\mathrm{S}, \mathrm{ES}\}$, when the matrices shown in Table IV (i.e., the ESs) are not known yet. On the contrary, NSE gives information about how the simulations are efficiently distributed among the different scenarios, once the matrices in Table IV (i.e., the ESs) begin to be known as a result of the preliminary exploration. The results of the preliminary guided exploration with $\gamma=1$ are compared to those of: (1) a crude Monte Carlo simulation method (MC) that randomly selects the scenario and, then, simulates the proper transition times according to the uniform sampling criterion proposed in Section 2; (2) an entropy-driven exploration, ${ }^{(25)}$ which follows a procedure similar to the guided exploration, but with an entropy-driven function instead of $I_{\gamma}(\cdot)$.

Figs. 9-11 show the empirical cumulative density functions (cdfs) of NFE (left) and NSE (right) built on 1,000 samples for the three configurations of the parameters considered in Table IV. It can be seen that the preliminary guided exploration with $\gamma=1$ achieves better or at least comparable performance than the entropy-driven exploration in all configurations considered, for both indexes. This is shown by the fact that the cdfs obtained by the guided

Table IV. Matrices of the End States that the System Can Reach for Each Scenario for Different Sets of Flow Rate Parameters Values: $\left[\phi_{a}, \phi_{b}, \phi_{c}\right]=[8,5,5] \cdot 10^{4} \mathrm{~m}^{3} /$ day (left); $\left[\phi_{a}, \phi_{b}, \phi_{c}\right]=[8,3.7,5] \cdot 10^{4} \mathrm{~m}^{3} /$ day (middle), and $\left[\phi_{a}, \phi_{b}, \phi_{c}\right]=[8,2.2,6] \cdot 10^{4} \mathrm{~m}^{3} /$ day (right)

\begin{tabular}{cccccccccccccccccccc}
\hline & $E S_{1}$ & $E S_{2}$ & $E S_{3}$ & $E S_{4}$ & $E S_{5}$ & $E S_{6}$ & $E S_{1}$ & $E S_{2}$ & $E S_{3}$ & $E S_{4}$ & $E S_{5}$ & $E S_{6}$ & & $E S_{1}$ & $E S_{2}$ & $E S_{3}$ & $E S_{4}$ & $E S_{5}$ & $E S_{6}$ \\
\hline$S_{1}$ & 0 & 0 & 0 & 0 & 0 & 1 & 0 & 0 & 0 & 0 & 0 & 1 & & 0 & 0 & 0 & 0 & 0 & 1 \\
$S_{2}$ & 1 & 0 & 0 & 1 & 0 & 1 & 1 & 0 & 0 & 1 & 0 & 1 & & 1 & 0 & 0 & 1 & 0 & 1 \\
$S_{3}$ & 0 & 0 & 1 & 0 & 1 & 1 & 0 & 1 & 1 & 1 & 1 & 1 & & 0 & 1 & 0 & 1 & 1 & 1 \\
$S_{4}$ & 1 & 1 & 1 & 1 & 1 & 1 & 1 & 1 & 1 & 1 & 1 & 1 & & 1 & 1 & 0 & 1 & 0 & 1 \\
$S_{5}$ & 1 & 1 & 1 & 1 & 1 & 1 & 1 & 1 & 1 & 1 & 1 & 1 & & 1 & 1 & 0 & 1 & 0 & 1 \\
$S_{6}$ & 0 & 0 & 1 & 0 & 1 & 1 & 0 & 0 & 1 & & 1 & 1 & & 0 & 0 & 1 & 0 & 1 & 1 \\
$S_{7}$ & 1 & 1 & 1 & 1 & 1 & 1 & 1 & 1 & 1 & 1 & 1 & 1 & & 1 & 1 & 1 & 1 & 1 & 1 \\
$S_{8}$ & 1 & 1 & 1 & 1 & 1 & 1 & 1 & 1 & 1 & 1 & 1 & 1 & & 1 & 1 & 1 & 1 & 1 & 1 \\
\hline
\end{tabular}



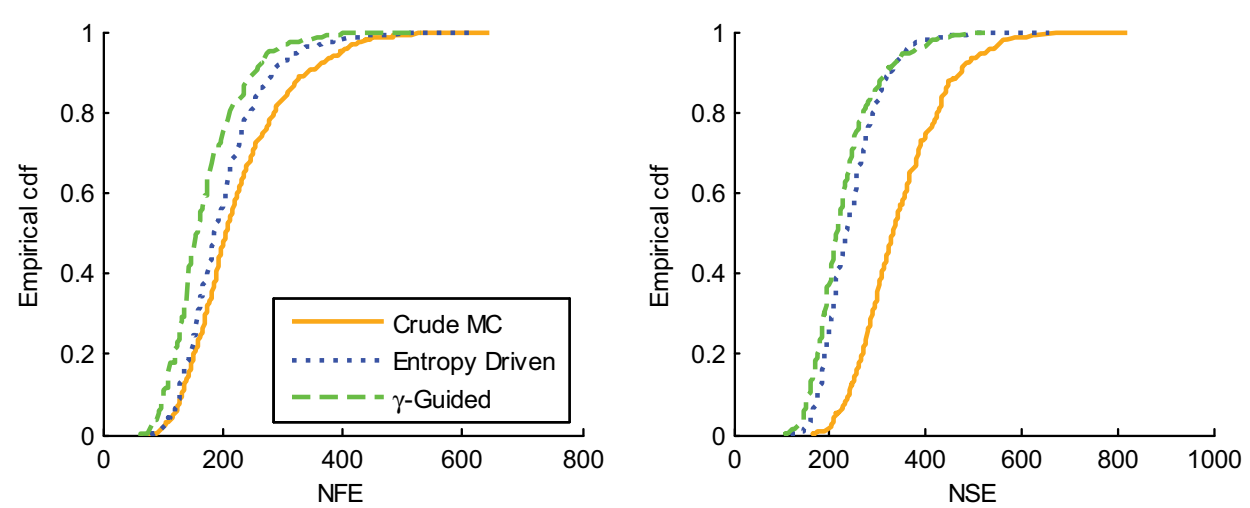

Fig. 9. Empirical cdfs of the NFE (left) and of the NSE (right) for crude MC (light line), for an entropy-driven method (dark dotted line), and for the preliminary guided exploration with $\gamma=1$ (light dashed line) with flow rate parameters $\left[\phi_{a}, \phi_{b}, \phi_{c}\right]=[8,5,5] \cdot 10^{4} \mathrm{~m}^{3} / \mathrm{day}$.
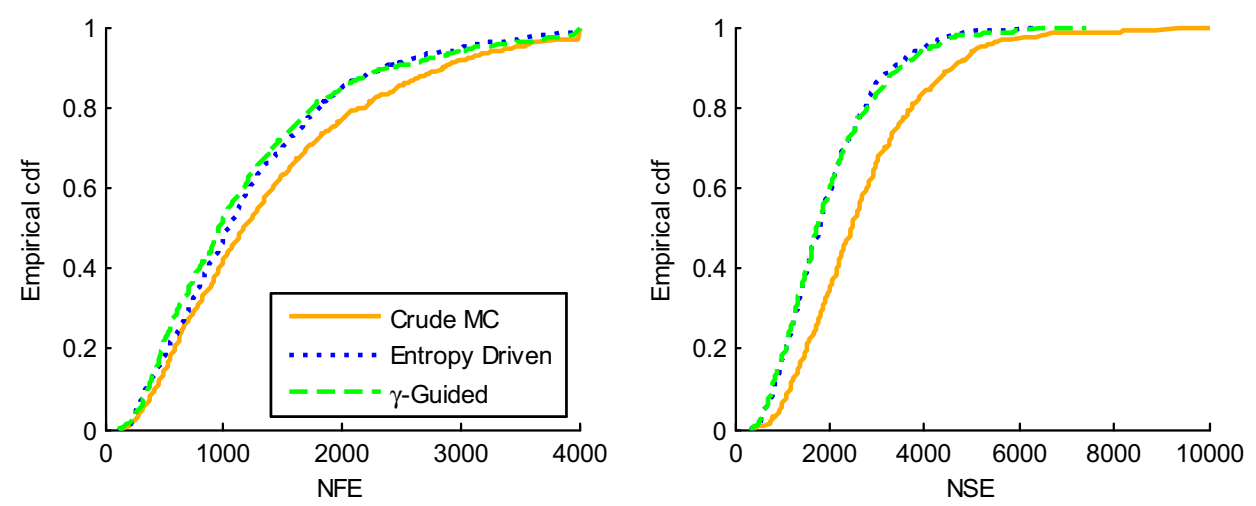

Fig. 10. Empirical cdfs of the NFE (left) and of the NSE (right) for crude MC (light line), for an entropy-driven method (dark dotted line), and for the preliminary guided exploration with $\gamma=1$ (light dashed line) with flow rate parameters $\left[\phi_{a}, \phi_{b}, \phi_{c}\right]=[8,3.7,5] \cdot 10^{4} \mathrm{~m}^{3} / \mathrm{day}$.
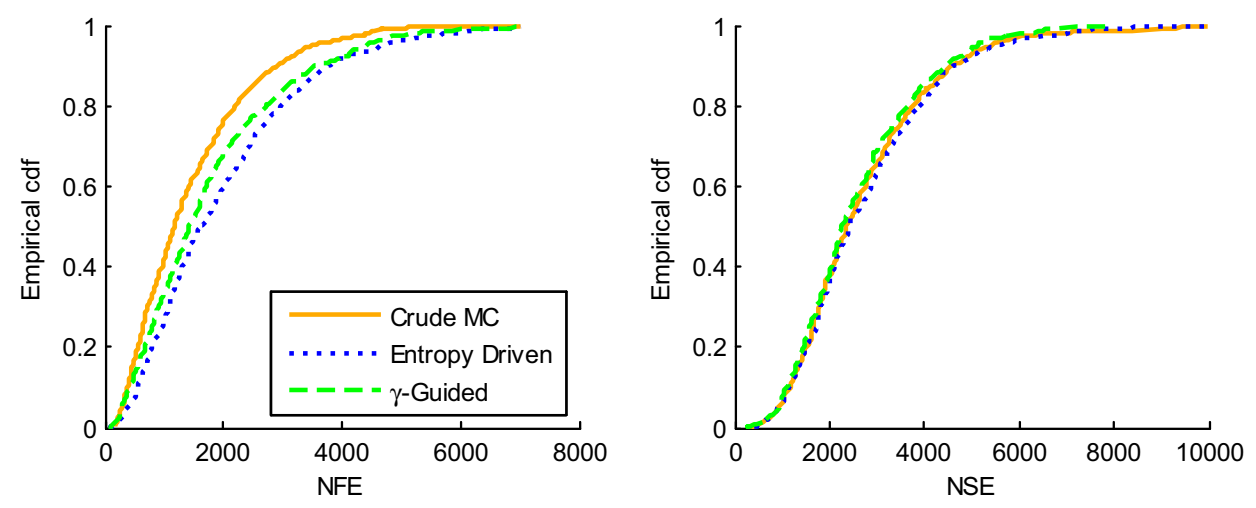

Fig. 11. Empirical cdfs of the NFE (left) and of the NSE (right) for crude MC (light line), for an entropy-driven method (dark dotted line), and for the preliminary guided exploration with $\gamma=1$ (light dashed line) with flow rate parameters $\left[\phi_{a}, \phi_{b}, \phi_{c}\right]=[8,2.2,6] \cdot 10^{4} \mathrm{~m}^{3} / \mathrm{day}$.

exploration (light dashed line) are "shifted" to the left with respect to the cdfs obtained by the entropydriven method (dark dotted line). Both these two steered methods ( $\gamma$-guided and entropy-driven) outperform the crude MC (light line) in the majority of the configurations of the parameters considered. However, the performance of the guided and of the entropy-driven explorations could be worse than that of the crude MC method for some configurations of the parameters (see, e.g., Fig. 11). Indeed, if the 


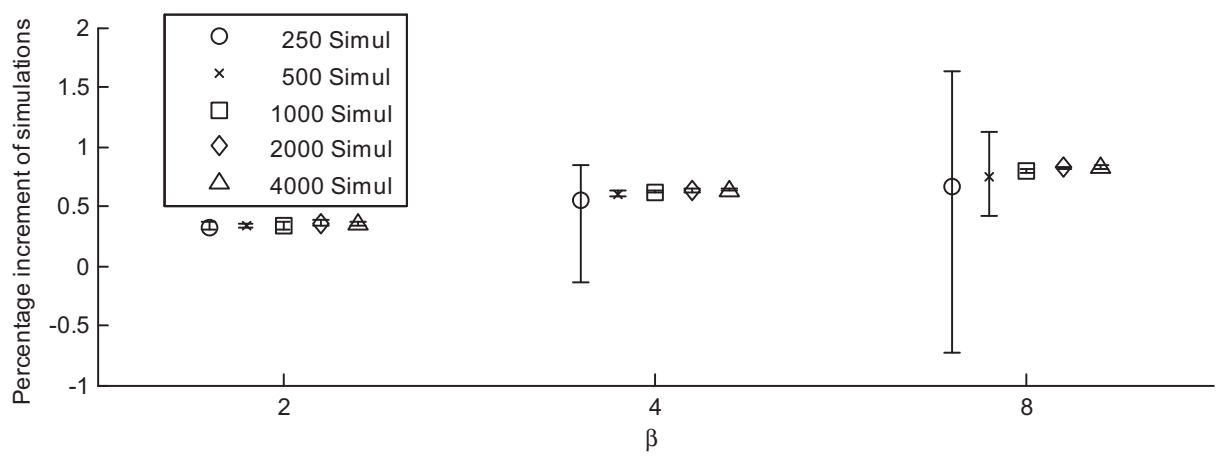

Fig. 12. Average percentage increment of simulations in a given scenario of interest $S_{7}$ and the respective percentile (10\% and $90 \%$ ), for parameter $\boldsymbol{\beta}=\{2 ; 4 ; 8\}$ and for different numbers of simulations.

configuration is such that the rarest event to visit is inside a scenario (e.g., $S_{4}, S_{5}$ in this case) that may reach a small number of ESs (i.e., only 4), then, using a method that increases the number of simulations in those scenarios that can reach a larger number of ESs is not effective. Nonetheless, while the entropydriven method is stuck, the guided exploration allows changing parameter $\gamma$ in order to increase the exploration effectiveness.

\subsection{Preliminary Forced Simulation}

For the analysis of the effects of the preliminary forced exploration, we have chosen the system configuration with flow rate parameter vector $\left[\phi_{\mathrm{a}}, \phi_{\mathrm{b}}, \phi_{\mathrm{c}}\right]=[8,2.2,6] \cdot 10^{4} \mathrm{~m}^{3} /$ day. In this setting, we assume to be interested in thoroughly exploring those scenarios that can lead to $E S_{3}$. The performance of the forced exploration is assessed by means of the average percent increment of simulations falling inside the scenarios of interest, i.e., those that can reach $E S_{3}$, with respect to the preliminary guided exploration. Different values of the parameter $\beta=\{2 ; 4 ; 8\}$ are considered to show the effects on the increment in the presence of different levels of computational resources available, i.e., with respect to different numbers of simulation runs $[250 ; 500 ; 1,000 ; 2,000 ; 4,000]$. Fig. 12 reports the average percent increment and the respective 0.1 and 0.9 percentiles on 1,000 experiments for each combination of the values of parameter $\beta$ and number of simulations, for a chosen scenario of interest $\left(S_{7}\right)$. According to the definition of the function in Equation (3), the larger the parameter $\beta$, the larger the average percentage increment of simulations in the scenario of interest, i.e., around $28 \%$ for $\beta=\{2 ; 4 ; 8\}$, respectively. However, it must be considered that if parameter $\beta$ is too large compared to the computational effort, then the performance can be more uncertain, as shown by the intervals corresponding to $\beta=8$ and a number of simulations lower than 500 . This is due to the fact that if the parameter $\beta$ is too large, then the forced exploration will focus its attention (i.e., the simulations) on the scenario that firstly discovers (i.e., reaches) the end state of interest (e.g., $E S_{3}$ ) before other scenarios can reach the end state of interest $\left(E S_{3}\right)$ as well. The larger the number of scenarios that can reach the ES of interest, the larger the sensitivity to the number of simulations, given $\beta$. We do not report the figures for the other two scenarios of interest $\left(S_{6}\right.$ and $\left.S_{8}\right)$, since they show similar results.

It must be pointed out that the average increment of simulations in the scenarios of interest is conditioned to the number of scenarios that can reach the end state of interest. Indeed, assuming that we are interested in exploring end state $E S_{6}$ (which can be reached by all scenarios), then no preference is given to any scenario since the forcing function equally increments the preference for all of them. Furthermore, since the information about the number of end states that a given scenario can reach is typically not available, the previous observation suggests that the forced scheme guarantees an exploration at least as effective as the guided one, when all scenarios can reach the end state of interest.

\subsection{Deep Exploration}

After a preliminary guided exploration of the system defined by parameters $\left[\phi_{a}, \phi_{b}, \phi_{c}\right]=$ $[8,3.6,5] \cdot 10^{4} \mathrm{~m}^{3} /$ day a large variability in the outcomes is observed within scenario $S_{5}$, as highlighted in Table V. Thus, it is interesting to retrieve the event 

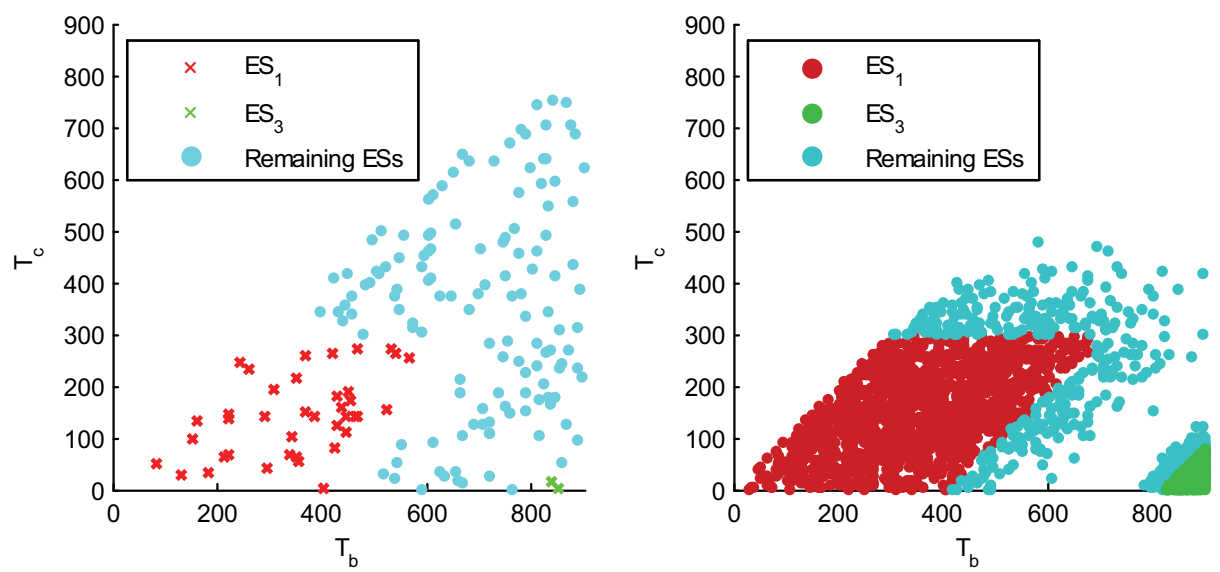

Fig. 13. Preliminary guided exploration of $S_{5}$ (left) and deep exploration of $\boldsymbol{E} \boldsymbol{S}_{1}$ and $\boldsymbol{E} \boldsymbol{S}_{3}$ in the same scenario (right).

Table V. Matrix Reporting the ESs Visited by a Preliminary Guided Exploration of the System with Parameters $\left[\phi_{a}, \phi_{b}, \phi_{c}\right]=[8,3.6,5] \cdot 10^{4} \mathrm{~m}^{3} /$ day, given a Computational Effort of 1,000 Simulations

\begin{tabular}{ccccccc}
\hline & $E S_{1}$ & $E S_{2}$ & $E S_{3}$ & $E S_{4}$ & $E S_{5}$ & $E S_{6}$ \\
\hline$S_{1}$ & 0 & 0 & 0 & 0 & 0 & 29 \\
$S_{2}$ & 21 & 0 & 0 & 38 & 0 & 28 \\
$S_{3}$ & 0 & 27 & 10 & 24 & 36 & 47 \\
$S_{4}$ & 46 & 29 & 0 & 41 & 5 & 23 \\
$S_{5}$ & 39 & 50 & 2 & 57 & 7 & 18 \\
$S_{6}$ & 0 & 0 & 23 & 0 & 28 & 36 \\
$S_{7}$ & 38 & 36 & 22 & 36 & 14 & 26 \\
$S_{8}$ & 34 & 39 & 24 & 41 & 12 & 22 \\
\hline
\end{tabular}

time sequences that lead to two chosen ESs: $E S_{1}$, which represents the worst final condition, and $E S_{3}$, which has been visited only few times during the preliminary exploration (e.g., according to Table V, highlighted row).

The space filling parameter is set to 0.2 with a maximum number of simulations to run set to 5,000 . The covariance matrix has been estimated from the vectors of transient times obtained from the preliminary exploration with respect to $E S_{1}$. On the contrary, since only two vectors are available for $E S_{3}$, an independent Gaussian proposal with standard deviation equal to the Euclidean distance between the two vectors is considered. The chosen standard deviation provides an idea of the dimension of the support to explore. Fig. 13 reports the transition time vectors of the scenario of interest $S_{5}$ after the preliminary exploration (on the left) and after the deep exploration (on the right). Results confirm that the proposed deep exploration is capable of in- creasing the number of simulations around the time sequences that reach the ES of interest, increasing the information about those sequences that can lead the system to a particular event. For example, in order to obtain $E S_{3}$, pipe c should break within the initial 100 days whereas pipe $b$ should work at least for 800 days after the failure of the first one.

\section{CONCLUSIONS}

IDPSA is expected to lead to a more realistic evaluation of the risks associated to safety-critical systems (e.g., nuclear power plants). One opportunity that is sought is the discovery and understanding of the possible outcomes from the dynamics of accidents, leaving out as little as possible of the unexpected. Thorough system state-space exploration in IDPSA allows identifying extreme situations and critical system characteristics for preventing accidents and/or mitigating their effects.

In this article, a novel framework for the exploration of the system state-space has been proposed. The framework allows including analyst prior knowledge about the system and focusing the exploration on particular configurations of interest, e.g., due to their criticality and/or rarity. Three methods, namely, guided, forced, and deep exploration, are implemented to allow diversifying the exploration in accordance with the preferences and interests of the analyst. Application to a simple system has shown that the proposed framework outperforms an entropy-driven method of the literature, ${ }^{(25)}$ as well as a crude Monte Carlo method, in exploring the system state-space. In addition, the deep exploration 
phase has been shown capable of leading a large number of simulations to the events of interest.

Future research efforts will be devoted to extending the exploration framework to the assessment of the probability of the events of interest and to applications to more complex case studies.

\section{REFERENCES}

1. Smidts C, Devooght J. Probabilistic reactor dynamics. 2. A Monte-Carlo study of a fast-reactor transient. Nuclear Science and Engineering, 1992; 111(3):241-256.

2. Gao LJ, Liu SY, Dougal RA. Dynamic lithium-ion battery model for system simulation. IEEE Transactions on Components and Packaging Technologies, 2002; 25(3):495-505.

3. Hansen AD, Jauch C, Sørensen PE, Iov F, Blaabjerg F. Dynamic wind turbine models in power system simulation tool digsilent. Roskilde, 2004; Ris $\varnothing$ National Laboratory; Ris $\varnothing-\mathrm{R}-1400(\mathrm{EN})$. Available at: http://orbit.dtu.dk/ fedora/objects/orbit:91193/datastreams/file_7711226/content.

4. Bier VM, Haimes YY, Lambert JH, Matalas NC, Zimmerman R. A survey of approaches for assessing and managing the risk of extremes. Risk Analysis, 1999; 19(1):83-94.

5. Pate-Cornell E. Finding and fixing systems weaknesses: Probabilistic methods and applications of engineering risk analysis. Risk Analysis, 2002; 22(2):319-334.

6. Paté-Cornell E. On "black swans" and "perfect storms": Risk analysis and management when statistics are not enough. Risk Analysis, 2012; 32(11):1823-1833.

7. Siu N. Risk assessment for dynamic-systems: An overview. Reliability Engineering \& System Safety, 1994; 43(1):43-73.

8. Li JH, Kang R, Mosleh A, Pan X. Simulation-based automatic generation of risk scenarios. Journal of Systems Engineering and Electronics, 2011; 22(3):437-444.

9. Aldemir T. A survey of dynamic methodologies for probabilistic safety assessment of nuclear power plants. Annals of Nuclear Energy, 2013; 52:113-124.

10. Cojazzi G. The DYLAM approach for the dynamic reliability analysis of systems. Reliability Engineering \& System Safety, 1996; 52(3):279-296.

11. Hsueh KS, Mosleh A. The development and application of the accident dynamic simulator for dynamic probabilistic risk assessment of nuclear power plants. Reliability Engineering \& System Safety, 1996; 52(3):297-314.

12. Labeau PE, Smidts C, Swaminathan S. Dynamic reliability: Towards an integrated platform for probabilistic risk assessment. Reliability Engineering \& System Safety, 2000; 68(3):219-254

13. Cepin M, Mavko B. A dynamic fault tree. Reliability Engineering \& System Safety, 2002; 75(1):83-91.

14. Kloos M, Peschke J. MCDET: A probabilistic dynamics method combining Monte Carlo simulation with the discrete dynamic event tree approach. Nuclear Science and Engineering, 2006; 153(2):137-156.

15. Hakobyan A, Aldemir T, Denning R, Dunagan S, Kunsman D, Rutt B, Catalyurek U. Dynamic generation of accident progression event trees. Nuclear Engineering and Design, 2008; 238(12):3457-3467.

16. Devooght J, Smidts C. Probabilistic reactor dynamics. 1. The theory of continuous event trees. Nuclear Science and Engineering, 1992; 111 (3):229-240.

17. Garrett C, Apostolakis G. Context in the risk assessment of digital systems. Risk Analysis, 1999; 19(1):23-32.
18. Di Maio F, Baronchelli S, Zio E. A computational framework for prime implicants identification in noncoherent dynamic systems. Risk Analysis, 2015; 35(1):142-156.

19. The RELAP5-3D Code Development Team, RELAP5-3D Code Manual Volume I: Code Structure, System Models and Solution Methods, INL-EXT-98-00834-V1, Revision 4.2, Idaho National Laboratory, June, 2014. Available at: http://www4vip.inl.gov/relap5/

20. Fong CJ, Apostolakis GE, Langewisch DR, Hejzlar P, Todreas NE, Driscoll MJ. Reliability analysis of a passive cooling system using a response surface with an application to the flexible conversion ratio reactor. Nuclear Engineering and Design, 2009; 239 (12):2660-2671.

21. Perez M, Reventos F, Batet L, et al. Uncertainty and sensitivity analysis of a LBLOCA in a PWR nuclear power plant: Results of the phase V of the BEMUSE programme. Nuclear Engineering and Design, 2011; 241(10):4206-4222.

22. Rutt B, Catalyurek U, Hakobyan A, Metzroth K, Aldemir T, Denning R, Dunagan S, Kunsman D. Distributed dynamic event tree generation for reliability and risk assessment. Challenges of Large Applications in Distributed Environments, Proceedings, 2006:61-70.

23. Zio E. Integrated deterministic and probabilistic safety assessment: Concepts, challenges, research directions. Nuclear Engineering and Design, 2014; 280:413-419.

24. Hu Y, Groen F, Mosleh A. An entropy-based exploration strategy in dynamic PRA. Pp. 2391-2397 in Probabilistic Safety Assessment and Management. Berlin: Springer, 2004.

25. Turati P, Pedroni N, Zio E. An entropy-driven method for exploring extreme and unexpected accident scenario in the risk assessment of dynamic engineered systems. Safety and Reliability of Complex Engineered Systems: ESREL 2015; CRC Press 2015; 761-769. DOI: 10.1201/b19094-102.

26. Mackay DJC. Information-based objective functions for active data selection. Neural Computation, 1992; 4(4):590604.

27. Hu Y. A Guided Simulation Methodology for Dynamic Probabilistic Risk Assessment of Complex Systems. College Park, MD: University of Maryland, 2005.

28. Cover TM, Thomas JA. Elements of Information Theory, 2nd ed. Hoboken, NJ: Wiley-Interscience, 2006.

29. Mercurio D, Podofillini L, Zio E, Dang VN. Identification and classification of dynamic event tree scenarios via possibilistic clustering: Application to a steam generator tube rupture event. Accident Analysis \& Prevention, 2009; 41(6):11801191.

30. Catalyurek U, Rutt B, Metzroth K, Hakobyan A, Aldemir T, Denning R, Dunagan S, Kunsman D. Development of a codeagnostic computational infrastructure for the dynamic generation of accident progression event trees. Reliability Engineering \& System Safety, 2010; 95(3):278-294.

31. Di Maio F, Vagnoli M, Zio E. Risk-based clustering for near misses identification in integrated deterministic and probabilistic safety analysis. Science and Technology of Nuclear Installations, 2015; 501:693891.

32. Robert CP, Casella G. Monte Carlo Statistical Methods, 2nd ed. New York: Springer, 2004.

33. Chib S, Greenberg E. Understanding the Metropolis-Hastings algorithm. American Statistician, 1995; 49(4):327-335.

34. Andrieu C, Thoms J. A tutorial on adaptive MCMC. Statistics and Computing, 2008; 18 (4):343-373.

35. Roberts GO, Rosenthal JS. Examples of adaptive MCMC. Journal of Computational and Graphical Statistics, 2009; 18(2):349-367. 